\title{
NON-MENDELIAN FEMALE STERILITY IN DROSOPHILA MELANOGASTER: HEREDITARY TRANSMISSION OF REACTIVITY LEVELS
}

\author{
ALAIN BUCHETON and GEORGES PICARD \\ Laboratoire de Génétique, Université de Clermont-Ferrand, B.P. 45, 63170
}

Aubière, France

Received 2.vi.77

\section{Summary}

Crosses between various strains of Drosophila melanogaster may give rise to a female sterility of non-Mendelian determination. When two factors, of as yet unknown nature, are contributed by the gametes from which $F_{1}$ females originate, these flies may show a more or less strongly reduced fertility. The factor carried by " reactive" strains ( $R$ factor) varies considerably in its ability to reduce fertility. This characteristic was used in experiments to analyse the heredity of $R$. It appears to be under complex control involving both extrachromosomal and classical nuclear inheritance.

\section{INTRODUCTION}

GRosses between various strains of Drosophila melanogaster give, in some cases, $F_{1}$ females showing a reduced fertility. On the basis of the fertility of the $F_{1}$ females, all strains can be assigned to three categories: reactive, inducer and neutral. The reduced fertility is observed in females, denoted $S F$ females, resulting from crosses between females of reactive strains and males of inducer strains. The reciprocal crosses and those involving neutral strains or within one category, give normally fertile females (Picard et al., 1972a).

From a survey of a large number of strains, it appeared that all those which arise from individuals recently collected in the wild are inducer, while there are laboratory strains of all three categories. Reactive and neutral strains thus seem to result from genetic changes which occur most readily in small isolates maintained in laboratory conditions (Picard et al., 1976). In connection with this, it may be noted that several authors (Rosenfeld et al., 1971; Sved, 1976; Kearsey et al., 1977; Kidwell et al., 1977) have reported cases of female sterility which seem to bear some similarity to our observations.

The reduced fertility of $S F$ females results from the fact that a certain proportion of the eggs they lay fail to hatch. The following features characterised this phenomenon (Picard et al., 1977):

1. Embryonic development is blocked at a precise stage, before the blastoderm stage.

2. Egg death is an entirely maternal characteristic. Its frequency does not depend on the males with which the $S F$ females are crossed and Mendelian segregations do not appear modified in the progeny of these females.

3. The level of sterility falls regularly with the aging of the $S F$ females.

4. The percentage of eggs that hatch can be reversibly increased by submitting the $S F$ females to a heat treatment. 
The sterility of $S F$ females may be looked upon as the result of an interaction between two factors of as yet unknown nature. The one brought to the $S F$ female by the reactive strain is called $R$ and the one which comes from the inducer strain $I$ (Picard et al., 1972b).

What was known about these factors before the experiments reported in the present paper amounted to the following points: the $I$ factor, when transmitted by males, has been shown to be linked strictly to inducer strain chromosomes. In contrast, in heterozygous females with chromosomes from both inducer and reactive strains, the chromosomes of reactive origin may acquire irreversibly the inducer character, independently of the production of recombined gametes. This phenomenon has been called chromosomal contamination (Picard, 1976).

Concerning the $R$ factor, the difference between reciprocal crosses of reactive and inducer strains suggests that $S F$ female sterility depends on the state of the cytoplasm in the oocytes of reactive females. This does not, however, provide any precise information about the genetic determination of this reactive state.

Both factors, $R$ and $I$, show great variability, the level of $S F$ female sterility depending, to a large extent, on the choice of the inducer and reactive parents. It is thus possible to define the level of reactivity of any reactive female by the reduction of fertility of the $S F$ daughters obtained after its cross with standard inducer males. A female is considered strongly reactive when the hatching percentage of eggs laid by its $S F$ daughters is low. It is considered weakly reactive when this hatching percentage is high (Bucheton et al., 1976). Some reactive strains are a mixture of strongly and weakly reactive flies, with all possible intermediates between the extreme types. From such strains, strongly and weakly reactive families, which breed respectively only strong or weak females, can be obtained either by selection or by spontaneous drift (Picard et al., 1972a; Bucheton, 1973).

Crosses between weak females and strong males lead to a majority of weak $F_{1}$ females and a minority of $F_{1}$ females of intermediate types. Reciprocal crosses lead to a majority of strong $F_{1}$ females and a minority of intermediate types. This suggests that, in the short term at least, the level of reactivity is transmitted chiefly by maternal inheritance but that the males also intervene in a minor way in this transmission (Bucheton, 1973).

The experiments described in this paper were undertaken to clear up the respective influences on the $S F$ female sterility of the cytoplasm and the maternal chromosomes, and also to study the infuence of the chromosomes in the inheritance of the levels of reactivity in reactive females.

\section{MATERIALS AND METHODS}

The genetic symbols are those used by Lindsley and Grell (1967). The flies are raised at $20^{\circ} \mathrm{C}$ on the axenic food described by David (1959).

\section{(i) Drosophila melanogaster stocks}

\section{(a) Inducer stocks}

Luminy $(L u)$ and $B 2^{\prime}$ are two wild type strains bred from flies captured in the wild, in 1969 in southern France and in 1972 in western France respectively. 
(b) Neutral stock

The neutral stock used in some experiments is the Paris strain which was bred from flies captured in Paris 30 years ago. This stock carries the cinnabar mutation.

\section{(c) Reactive stocks}

$s e F_{5}$ is a strong reactive family, established by selection by the method described by Picard et al. (1972a) from a strain homozygous for the mutation sepia.

$e$ is a weak reactive strain, homozygous for the mutation ebony.

$L H$ has the genotype In (1) $s c^{S 1} L_{s c^{8 R+S},} s c^{S 1} s c^{8} w^{a} B / \operatorname{In}(1) s c^{S 1 L_{s c}} c^{8 R+S}$, $s c{ }^{S 1}{ }^{8} c^{8} w^{a} B ; \operatorname{In}(2 L+2 R) C y / P m ; H / S b$. For the sake of clarity the $X$ chromosome of this stock will be referred to as $M-5(=$ Muller $5=$ Basc $)$ and the chromosome In $(2 L+2 R) C y$ by $C y$. This stock contains a mixture of strong, weak and intermediate type females.

$M-5-(R)$ is a weakly reactive strain with the genotype $\operatorname{In}(1) s c^{S 1} L_{S c}{ }^{8 R+S}$,

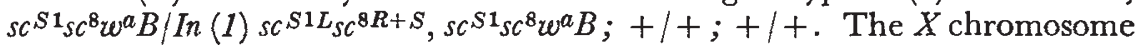
is referred to as $M-5$. This strain was obtained from The High Technical School of Agriculture, Valencia, Spain, and is referenced in Dros. Inf. Serv., 48 (1972), page 12.

$C y / B 1 L-(R)$ is a weakly reactive strain of genotype $+1+; \operatorname{In}(2 L+2 R)$ $C y / B 1 L ;+/+$. The chromosome $\operatorname{In}(2 L+2 R) C y$ is referred to as $C y$.

$D c x F / D f d-(R)$ is a weakly reactive strain of genotype $+/+;+1+$; In $(3 L R) D c x F / D f d$. The chromosome $\operatorname{In}(3 L R) D c x F$ is referred to as $D c x F$.

The $M-5, C y$ and $D c x F$ chromosomes from these stocks carry inversions and are used in crosses as crossing-over inhibitors.

\section{(ii) Measurement of the hatching percentages of eggs}

The females, the fertility of which is to be measured, are placed, two days after emergence, in culture tubes containing axenic food to which carbon black is added. When the fertility of individual females is to be measured, eggs are collected during about 48 hours. When, in other experiments, the overall fertility of a group of females is determined, eggs are collected during 24 hours. In all cases hatched and non-hatched eggs are counted 48 hours after the end of laying and a hatching percentage is determined.

The females whose fertility is studied are crossed either with their brothers or with wild type males of any strain; this choice does not affect the percentage of eggs that succeed to hatch (Picard et al., 1977).

\section{Results}

\section{(i) Influence of the maternal chromosomes of $\mathrm{SF}$ females on their sterility}

Crosses between reactive and inducer strains give rise to sterile offspring only when reactive females are crossed with inducer males. This difference between reciprocal crosses suggests that the sterility of an $F_{1}$ female depends on the origin of the cytoplasm of the egg from which it has evolved. This leaves open the question whether the particular maternal chromosomes which are inherited with the proper cytoplasm by a $S F$ female intervene in 
the determination of its level of sterility. An experiment, represented in fig. la, was carried out in order to try to clear up this point.

Go

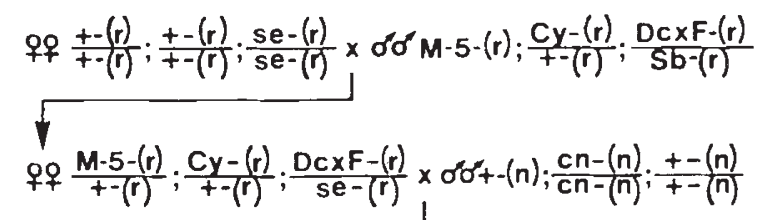

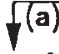

G2

$+\circ \frac{M-5-(r)}{+-(n)} ; \frac{C y-(r)}{C n-(n)} ; \frac{D c x F-(r)}{t-(n)}$

$\sigma^{x}+-(i) ; \frac{+-(i)}{+-(i)} ; \frac{+-(i)}{+-(i)}$

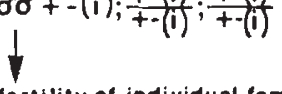

fertility of individual females

of the 8 genotypic classes.

G3 (b)

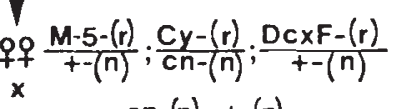

1 of $\sigma^{\prime}+-(n) ; \frac{c n-(n)}{c n-(n)} ; \frac{t-(n)}{+-(n)}$

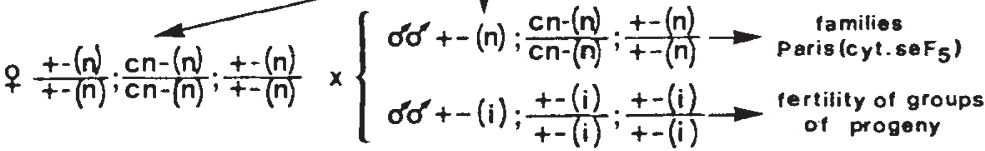

FIG. 1.- Influence of the maternal chromosomes of $S F$ females on their level of sterility (a) and effect on the level of reactivity of changing the genotype within the same maternal line (b): experimental plan. The G0 females come from the strongly reactive strain ${ }_{s e} F_{5}$ and the G0 males from a cross between the reactive strains $L H$ of genotype $M-5-(r) / M-5-(r) ; \quad C y-(r) / P m-(r) ; \quad H-(r) / S b-(r), \quad$ and $\quad D c x F / D f d-(R) \quad$ of genotype $+-(r) /+-(r) ;+-(r) /+-(r) ; D c x F-(r) / D f d-(r)$. The $+-(n)$ and $c n-(n)$ chromosomes come from the neutral strain Paris and the $+-(i)$ chromosomes from the inducer strain $L u$.

The initial crosses were made to provide generation 2 females which derive maternally from the strongly reactive strain $s e F_{5}$ and carry a halfgenome originating from the neutral strain Paris and a half-genome composed of the three major chromosomes of reactive origin marked by dominant genes associated with inversion complexes.

These females, with the genotype $M-5-(r) /+-(n) ; C y-(r) / c n-(n)$; $D c x F-(r) /+-(n)^{*}$ were crossed with males of the inducer strain $L u$, and the fertilities of 20 to 30 daughters belonging to each of the eight genotypic categories were individually measured.

The results (fig. 2) indicate that the $M-5-(r) /+-(n) ; C y-(r) / c n-(n)$; $D c x F-(r) /+-(n)$ females behave like reactive females since, when crossed with inducer males, they produce some more or less sterile daughters. However, this sterility does not appear to be linked to the segregation of the three major chromosomes. Many females, with the genotype $+-(n) /+-(i)$; $c n-(n) /+-(i) ;+-(n) /+-(i)$, which have only chromosomes of neutral origin, with the possible exception of chromosome 4, which is not controlled, are, on the average, much less fertile than females coming from crosses of Paris females with $L u$ males. The latter show high fertility (see Fig. 7c).

However, the eight distributions are not identical. A test of homogeneity was made between them. In this test, females were grouped into a number of classes according to their fertility, in such a way that the

* The chromosomes from inducer, neutral and reactive strains are indicated by the respective indices $-(i),-(n)$ and $-(r)$. 


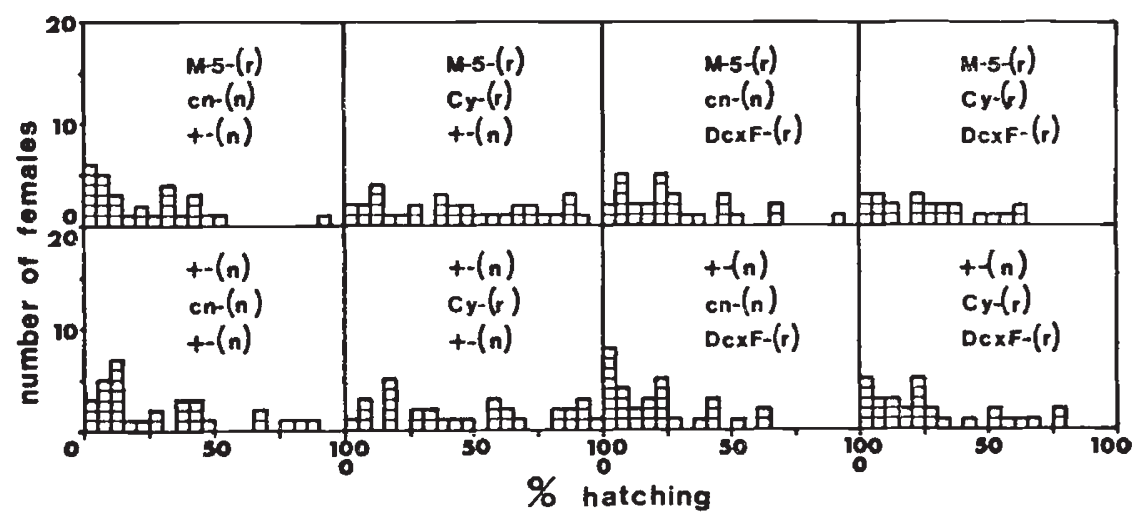

FIG. 2.-Influence of the maternal chromosomes of $S F$ females on their sterility. Each rectangle represents the level of fertility of a female descending from a mass cross of $M-5-(r) /+-(n) ; C y-(r) / c n-(n) ; D c x F-(r) /+-(n)$ females and males of the inducer strain $L u$. Each histogram represents the fertility of females belonging to one of the eight genotypic classes obtained. The half-genome of paternal origin was identical for all these females, and only the half-genomes of maternal origin are marked.

theoretical number in each class was greater than 5. Four classes were thus defined: 0 to 15 per cent, 15 to 30 per cent, 30 to 50 per cent and 50 to 100 per cent. The $\chi^{2}$ obtained is 39.48 (d.f. $=21 ; 0.001<\mathrm{P}<0.01$ ). A homogeneity test comparing the distribution obtained by grouping the females carrying the $X$ chromosome $M-5-(r)$ and the distribution obtained by grouping those with the $X$ chromosome $+-(n)$ indicate that the segregation of the $X$ chromosome is not responsible for the variations observed between the eight categories $\left(\chi^{2}=2.75\right.$; d.f. $\left.=3 ; 0.30<\mathrm{P}<0.50\right)$. The same operation made to study the influences of chromosomes 2 and 3 gave contradictory results. Females carrying chromosome $2 C y-(r)$ are significantly more fertile than those with chromosome $2 \mathrm{cn}-(n)\left(\chi^{2}=17 \cdot 24\right.$; d.f. $=3 ; \mathrm{P}<0.001)$, while those with chromosome $3 D c x F-(r)$ are significantly more sterile than those with chromosome $3+-(n)\left(\chi^{2}=11 \cdot 10\right.$; d.f. $=3 ; 0.01<\mathrm{P}<0.02)$. Finally, the distribution of females carrying the three chromosomes of reactive origin $M-5-(r), C y-(r)$ and $D c x F-(r)$ is not different from that of females carrying the three chromosomes of neutral origin $\left(\chi^{2}=1.13\right.$; d.f. $\left.=3 ; 0.70<\mathrm{P}<0.80\right)$. In spite of the differences observed between the eight distributions, it does not appear that the $S F$ females which have inherited from their mothers chromosomes of reactive origin show a more reduced fertility than those which have inherited the homologous chromosomes of neutral origin.

The segregation of chromosome 4 does not appear to play an important

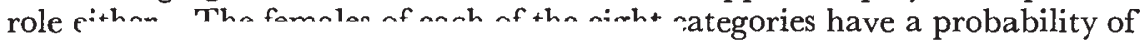
0.5 of al strain Paris. If the chromosome cams of fig. 2 , half the females should, statistically, show a high fertility, and this is not at all the case.

Thus, the amount of reduction of fertility of $S F$ females does not depend, or depends to a quite minor extent, on the reactive or neutral origin of the chromosomes they received from their mothers. This fact is corroborated by another experiment which is not reported in this paper (Picard, 1977). The sterility of $S F$ females appears to be determined by a specific cytoplasmic 
state in the oocytes of the reactive females from which they originated. This leaves open the question whether reactivity in reactive strains is quite independent of the chromosomes and follows a true extrachromosomal inheritance or whether it is the result of a maternal effect.

\section{(ii) Influence of the paternal chromosomes in the determination of the levels of reactivity of reactive females}

As reported in the introduction, the level of reactivity cannot be a purely maternally transmitted character, since, in the crosses between reactive strains, males intervene in the determination of the level of reactivity of their daughters (Bucheton, 1973). Several experiments were carried out in order to study the influence of the chromosomes which are carried by the spermatozoa.

Males with the genotype $+-(r w) ; C y-(r w) /+-(r s) ; D c x F-(r w) / s e-(r s)^{*}$ were obtained from the strains $s e F_{5}, M-5-(R), C y / B 1 L-(R)$ and $D c x F / D f d-(R)$, by the crosses schematised in fig. $3 \mathrm{~b}$. The other crosses are given in fig. $3 \mathrm{c}$.

These males were crossed with females from the strong reactive strain $s e F_{5}$. Daughters of the four genotypic classes were then collected among the progeny of this cross and their levels of reactivity were measured. For this purpose, about 50 females of each genotypic class were isolated and crossed with inducer males from the strain $B 2^{\prime}$. Among the offspring of each individual cross, some wild type individuals were selected and their fertility was measured.

In order to test the influence of the $X$ chromosome on levels of reactivity, another series of crosses was made (fig. 3d). From the cross 우 $s e F_{5} \times \widehat{o} \sigma^{t}+-(r w) ; C y-(r w) /+-(r s) ; D c x F-(r w) / s e-(r s)$, males of the genotype $+-(r s) ; C y-(r w) /+-(r s) ; D c x F-(r w) / s e-(r s)$ were collected and crossed with $s e F_{5}$ females. The level of reactivity of the females of each of the four genotypic categories obtained was determined as before.

These experiments thus provided measurements of the levels of reactivity of females all derived in maternal line from the strongly reactive strain $\mathrm{seF}_{5}$ and carrying in heterozygous state either none or one or more chromosomes from weakly reactive strains.

As a control, the level of reactivity of $s e F_{5}$ females was determined by crossing them individually with males from the inducer strain $B 2^{\prime}$ and measuring the fertility of a group of $S F$ progeny for each cross. The level of reactivity of $M-5-\langle r w) /+-(r w) ; C y-(r w) /+-\langle r w) ; D c x F-(r w) /+-(r w)$ females obtained from the strains $M-5-(R), C y / B 1 L-(R)$ and $D c x F / D f d-(R)$ by the experimental procedure described in fig. $3 \mathrm{a}$, was also determined by the fertility of groups of wild type $S F$ daughters originating from individual crosses between these females and $B 2^{\prime}$ males.

The results are given as histograms in fig. $4 . s e F_{5}$ females are strongly reactive, for, when crossed with inducer males, they give almost entirely sterile $S F$ progeny (fig. 4a). In contrast, $M-5-(r w) /+-(r w) ; C y-(r w) /+-(r w)$; $D c x F-(r w) /+-(r w)$ females, arising from crosses between weakly reactive strains, are weakly reactive. When crossed with inducer males they give $S F$ daughters which are only slightly sterile (fig. 4 b).

* The chromosomes from strongly and weakly reactive strains are symbolised by indices $-(r s)$ and $-(r w)$ respectively. 


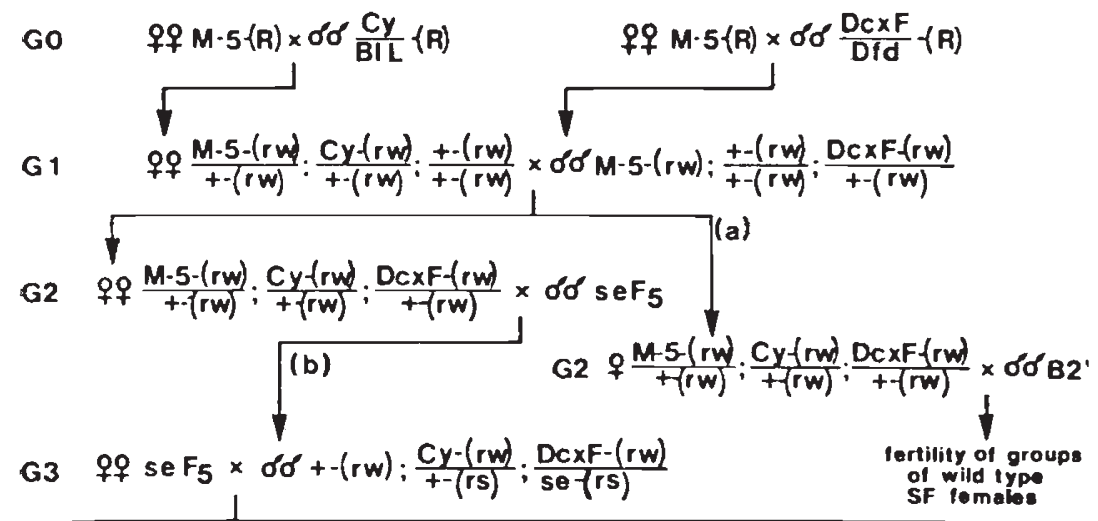

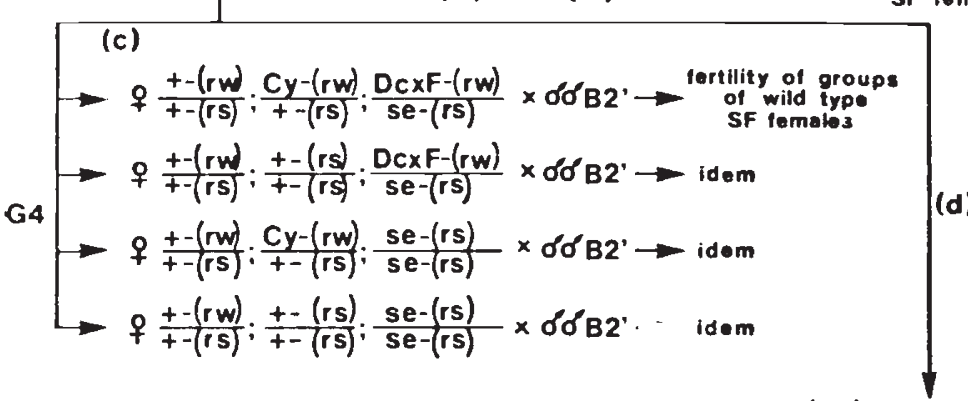

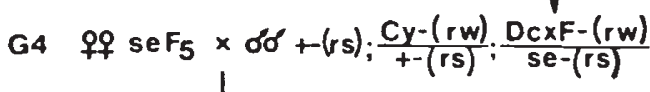

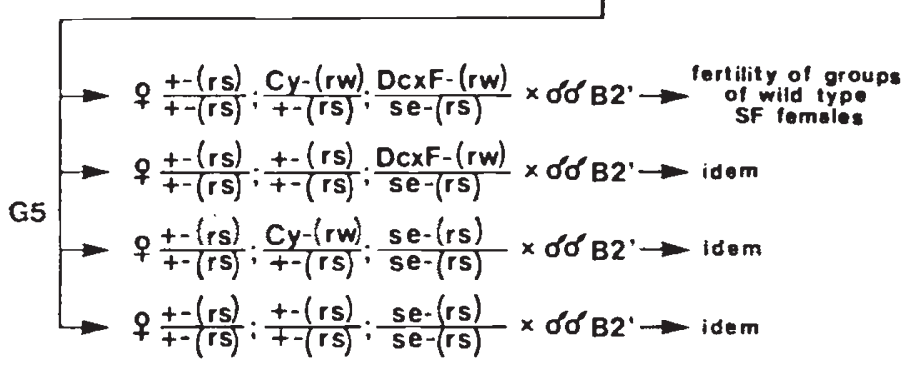

FIG. 3.-Constitution, in strongly reactive maternal line, of females carrying different combinations of chromosomes from strongly and weakly reactive strains. The level of reactivity of individual females of this type was measured, after crossing them with inducer $B 2^{\prime}$ males, by the hatching percentage of eggs laid by a group of their wild type $S F$ daughters (see text).

Most of the females in the eight genotypic classes behave as strongly reactive (fig. $4 \mathrm{c}$ to $\mathrm{j}$ ). They display a level of reactivity very different from that of $M-5-(r w) /+-(r w) ; C y-(r w) /+-(r w) ; D c x F-(r w) /+-(r w)$ females (fig. $4 \mathrm{~b}$ ) and more similar with that of the $s e F_{5}$ females (fig. $4 \mathrm{a}$ ) from which they descend in maternal line. However, differences between them exist in certain cases.

A homogeneity test was made to compare the level of reactivity of $+-(r s) /+-(r s) ;+-(r s) /+-(r s) ; s e-(r s) / s e-(r s)$ females (fig. $4 \mathrm{j})$, which, with the exception of chromosome 4 , reconstitute the genotype of the $s e F_{5}$ strain, with that of females of the $s e F_{5}$ strain (fig. $4 \mathrm{a}$ ). To this end, the reactive 


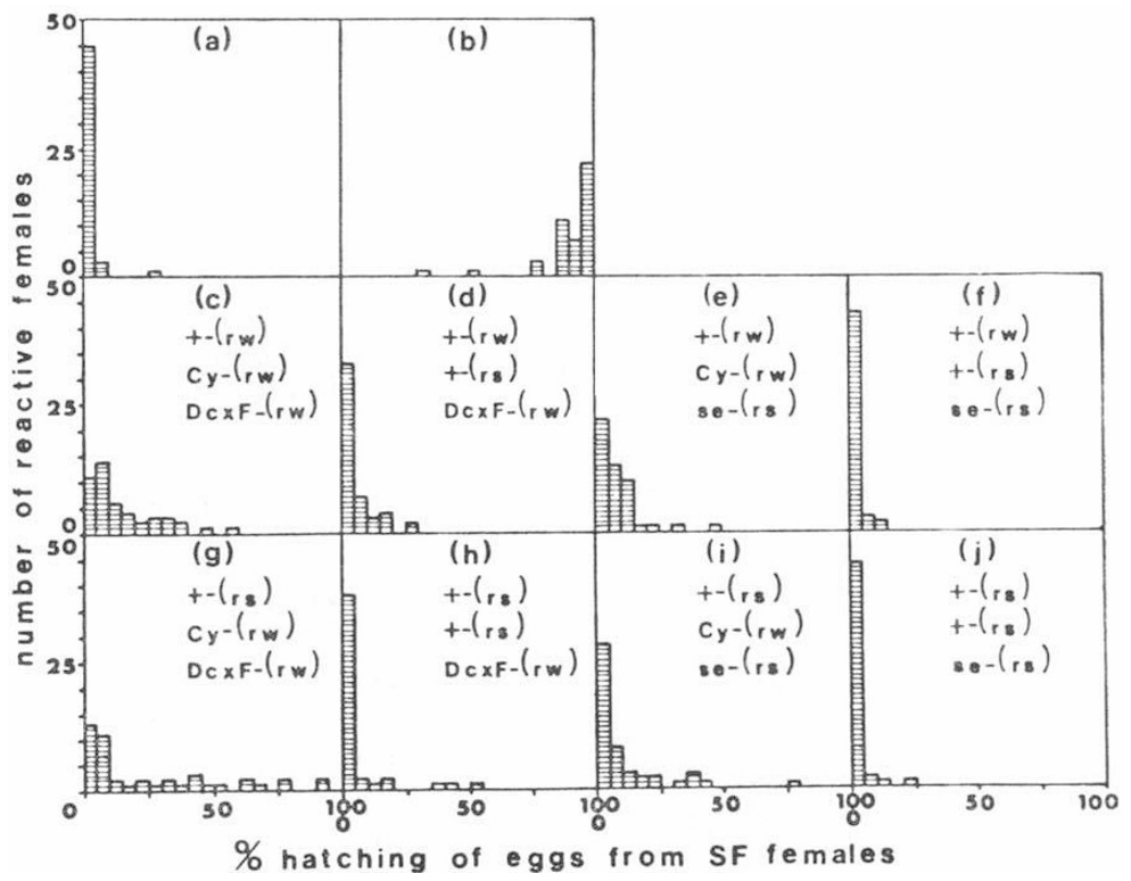

FIG. 4.--Levels of reactivity of females carrying different combinations of chromosomes from strongly and weakly reactive strains, obtained in strongly reactive maternal line. Each rectangle represents the level of reactivity of a single female, that is the overall fertility of a group of its wild type $S F$ daughters obtained after crossing it with $B 2^{\prime}$ inducer males.

(a) Reactivity of females of the strongly reactive strain $s e F_{5}$.

(b) Reactivity of females of genotype $M-5-(r w) /+-(n w) ; C y-(n w) /+-(r w)$; $D c x F-(r w) /+-(r w)$ obtained from weakly reactive strains $M-5-(R), C y / B 1 L-(R)$ and $D c x F / D f d-(R)$ (see fig. 3a).

(c) to (j) Reactivity of females carrying different combinations of chromosomes from strongly and weakly reactive strains, obtained in strongly reactive maternal line (see fig. 3). The females of each genotypic class all carry a half-genome from the strain $s e F_{5}$. This is not indicated in the histograms, and only the chromosomes of paternal origin of these females are shown.

females were divided into two classes according to the fertility of their $S F$ daughters (see table 1). The value of $\chi^{2}$ is 0.34 (d.f. $=1 ; P=0.37$ ), indicating that the two distributions are not significantly different.

The same operation made to compare the level of reactivity of $+-(r w) /+-(r s) ;+-(r s) /+-(r s) ; s e-(r s) / s e-(e s)$ females (fig. 4f) with that of females of the $s e F_{5}$ strain (fig. 4 a) leads to the same conclusion $\left(\chi^{2}=0.34\right.$; d.f. $=1 ; P=0 \cdot 37$ ). Since about half of the females of fig. $4 \mathrm{f}$ must carry, in heterozygous state, a chromosome 4 coming from a weak strain, this chromosome does not appear involved in the control of the levels of reactivity.

In contrast, the females of the six other genotypic classes show different reactivity compared with the $s e F_{5}$ females. The majority of these females are identical to their $\mathrm{SeF}_{5}$ mothers for reactivity, but in each genotypic category, a variable number of females are less reactive (fig. $4 c, d, e, g, h, i)$. Independently of the origin of their $X$ and fourth chromosomes, these females possess, in heterozygous state, either a chromosome 2 (fig. 4e and i), or a 
TABLE 1

Influence on the level of reactivity of chromosomes from weakly reactive strains (see also fig. 4). The reactive females were divided into two classes according to the fertility of their SF daughters: 1 , hatching percentages of eggs from SF females from 0 to 10 per cent; 2, hatching percentages from 10 to 100 per cent. The number of reactive females was standardised to 47 for each genotypic class ( fig. 4c to $j$ ). The value of the total homogeneity $\chi^{2}$ for the eight distributions is 52.00 $\left(d . f_{.}=7 ; P<0.001\right)$. The data forming an orthogonal set, this $\chi^{2}$ has been divided into its seven components. For each particular $\chi^{2}$, the number of degrees of freedom is 1

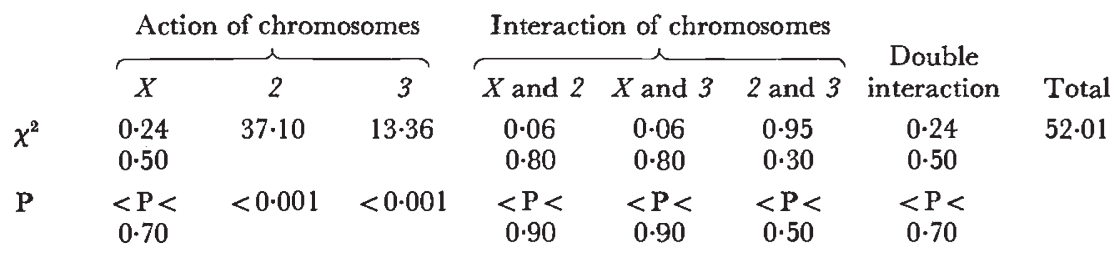

chromosome 3 (fig. $4 \mathrm{~d}$ and h), or both (fig. $4 \mathrm{c}$ and g), coming from weakly reactive strains.

Table 1 gives the results of a statistical test made to study the influence of the various chromosomes on the level of reactivity. It appears, firstly, that the $+-(r w) X$ chromosome has no effect. In contrast, the $C y$ - $(r w)$ chromosome and, to a minor extent, the $D c x F$ - $(r w)$ chromosome, have a significant influence on the levels of reactivity. The values obtained for the interactions are not significant, showing that the action of these chromosomes is additive.

In addition, this experiment shows that the differences observed between the females of different genotypic classes depend entirely on chromosomes and are not caused by some factor which might be brought by the spermatozoa independently of the chromosomes. Indeed, in fig. 4, females of four classes (histograms (c) to (f)) differ from females of the other four (histograms ( $\mathrm{g}$ ) to $(\mathrm{j}))$ not only by their $X$ chromosomes, but also by the maternal origin of their fathers. Those of the histograms (c) to (f) carry, in heterozygous state, an $X$ chromosome from a weakly reactive strain and arise from males obtained in maternal line from weakly reactive females (fig. $3 \mathrm{~b}$ and $\mathrm{c}$ ). Those of the histograms $(\mathrm{g})$ to $(\mathrm{j})$ carry $X$ chromosomes from the strongly reactive strain $s e F_{5}$ and arise from males obtained in maternal line from this same strain (fig. $3 \mathrm{~d}$ ). Table 1 indicates that the $+-($ rev $) X$ chromosome is not involved in the control of levels of reactivity, and therefore shows also that no modification in the levels of reactivity occurs by the action of a hypothetical cytoplasmic factor which might be brought by the spermatozoon and might be different according to the maternal origin of the male.

From this experiment, it may be concluded that, in crosses between females from strongly reactive strains and males from weak ones, the level of reactivity is chiefly transmitted maternally, since the majority of the females in the eight genotypic classes are as strongly reactive as their $s e F_{5}$ mothers. However, the $C y-(r w)$ and $D c x F-(r w)$ chromosomes from weak strains are able to modify partially the level of reactivity of the females that inherited them from their fathers.

Another similar experiment was carried out to determine whether paternal chromosomes from a strongly reactive strain may act in a parallel way. A diagram of the crosses is given in fig. 5 . 


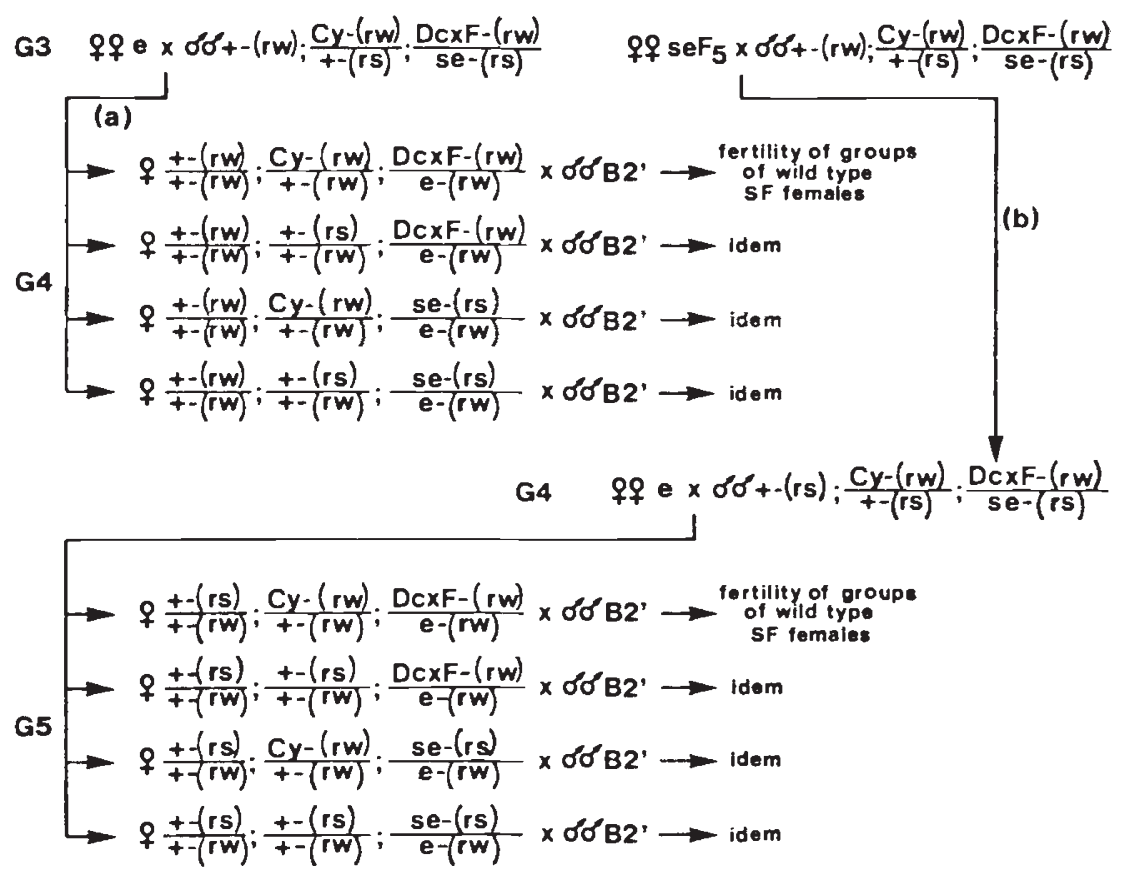

FIG. 5.-Constitution, in weakly reactive maternal line, of females carrying different combinations of chromosomes from strongly and weakly reactive strains. The level of reactivity of single females of this type was measured, after crossing them with B2' $^{\prime}$ inducer males, by the hatching percentage of eggs laid by a group of their wild type $S F$ daughters (see text).

(a) Constitution of females carrying $X$ chromosomes from weakly reactive strains.

(b) Constitution of females carrying, in heterozygous state, a chromosome $X$ from the strong reactive strain $s e F_{5}$.

Males of the genotype $+-(r w) ; C y-(r w) /+-(r s) ; D c x F-(r w) / s e-(r s)$ of the G3 generation were obtained from the strains $s e F_{5}, M-5-(R), C y / B 1 L-(R)$ and $D c x F / D f d-(R)$ by the same experimental procedure as described in fig. $3 \mathrm{~b}$.

These males were crossed with females from the weakly reactive strain $e$ (fig. 5a). Daughters from the four genotypic classes were then collected in the progeny of this cross and their reactivity was measured. To this end, about 50 females of each class were crossed individually with $B 2^{\prime}$ inducer males and the fertility of a set of their wild type $S F$ offspring was measured.

In order to test for the influence of the $X$ chromosome, males of the genotype $+-(r s) ; C y-(r w) /+-(r s) ; D c x F_{-}(r w) / s e-(r s)$, obtained from crosses between $s e F_{5}$ females and $+-(r w) ; C y-(r w) /+-(r s) ; D c x F-(r w) / s e-(r s)$ males, were crossed with $e$ strain females (fig. $5 \mathrm{~b}$ ). As before, the reactivity of the females of the four genotypic classes resulting from this cross was essayed by measuring the fertility of sets of wild type $S F$ daughters obtained after individual crosses of these females with $B 2^{\prime}$ males.

As controls, the reactivity of $e$ and $s e F_{5}$ females was measured, in addition to that of females with the genotype $M-5-(r w) /+-(r w) ; C y-(r w) /+-(r w)$; $D c x F-(r w) /+-(r w)$, which were obtained from the strains $M-5-(R), C y / B 1 L-(R)$ 
and $D c x F / D f d-(R)$ by an experimental procedure similar to the one described in fig. 3a. In each case, the $B 2^{\prime}$ strain was used as inducer.

The results are given as histograms in fig. 6. They confirm that the $s e F_{5}$ females are strongly reactive (fig. $6 \mathrm{~b})$, while the $M-5-(r w) /+-(r w)$; $C y-(r w) /+-(r w) ; D c x F-(r w) /+-(r w)$ females are weak (fig. 6c). The $e$ strain

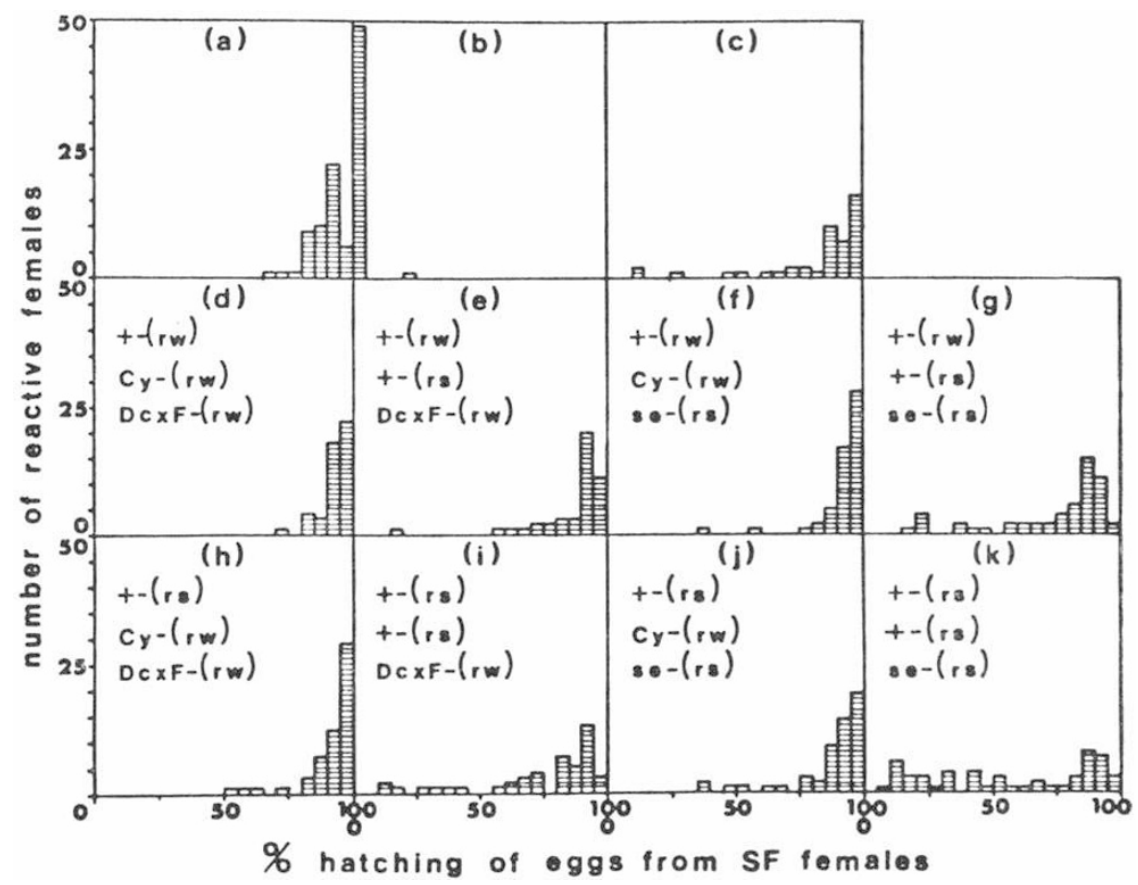

Fig. 6.-Levels of reactivity of females carrying different combinations of chromosomes from strongly and weakly reactive strains, obtained in weakly reactive maternal line. Each rectangle represents the level of reactivity of a single female, i.e. the overall fertility of a group of its wild type $S F$ daughters obtained after crossing it with $B 2^{\prime}$ inducer males.

(a) Reactivity of females of the weakly reactive strain $e$.

(b) Reactivity of females of the strongly reactive strain $s e F_{5}$.

(c) Reactivity of $M-5-(r w) /+-(r w) ; C y-(r w) /+-(r w) ; D c x F-(r w) /+-(r w)$ females obtained from the weakly reactive strains $M-5-(R), C y / B 1 L-(R)$ and $D c x F / D f d-(R)$ (experimental procedure identical as in fig. $3 \mathrm{a}$ ).

(d) to (k) Reactivity of females carrying different combinations of chromosomes from strongly and weakly reactive strains, obtained in weakly reactive maternal line (see fig. 5). The females of each genotypic class all carrying a half-genome from the $e$ strain, this is not indicated on the histograms. Only the chromosomes of paternal origin of these females are given.

females, which, after crossing with $B 2^{\prime}$ males, give only slightly sterile $S F$ progeny, are also weakly reactive (fig. $6 \mathrm{a}$ ).

Most of the females in the eight other genotypic categories behave as weakly reactive females (fig. $6 \mathrm{~d}$ to $\mathrm{k}$ ). Overall, they show very different reactivity from that of the $s e F_{5}$ females, and are similar, in most cases, to the $e$ strain females.

Table 2 gives the results of a statistical test made to study the influence of the various chromosomes coming from the $s e F_{5}$ strain on the level of 
TABLE 2

Influence on the level of reactivity of chromosomes from a strongly reactive strain (see also fig. 6). The reactive females were divided into two classes according to the fertility of their SF daughters: 1, hatching percentages of eggs from SF females from 0 to 85 per cent; 2, hatching percentages from 85 to 100 per cent. The number of reactive females was standardised to 50 for each genotypic class ( fig. $6 d$ to $k)$. The value of the total homogeneity $\chi^{2}$ for the eight distributions is $80.59(d . f .=7$; $P<0.001$ ). The data forming an orthogonal set, this $\chi^{2}$ has been divided into its seven components. For each particular $\chi^{2}$, the number of degrees of freedom is 1

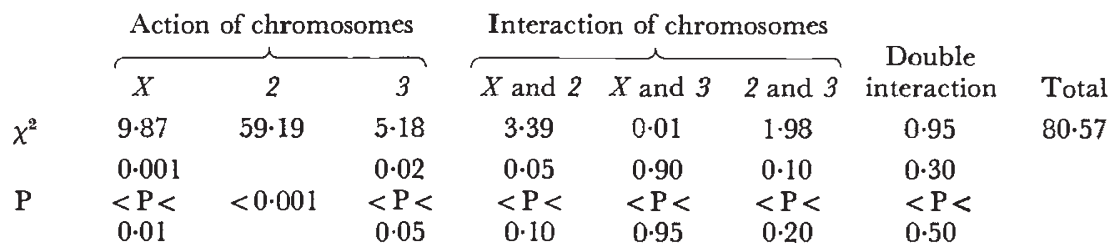

reactivity. It clearly shows that the $s e F_{5}$ chromosome 2 is able to modify largely the reactivity. The same is true for the $\mathrm{seF}_{5}$ chromosome $X$. However, this action is less powerful than that of the chromosome 2. The influence of the $s e F_{5}$ chromosome 3 is significant, but appears to be small. The values obtained for the interactions being not significant, it may be concluded that the three major chromosomes from the $s e F_{5}$ strain are additive in their action.

Thus, the results of this experiment confirm that the level of reactivity is chiefly transmitted maternally, since the majority of the females in the eight genotypic classes have a reactivity similar to that of their $e$ mothers. However, the chromosomes $X, 2$ and 3 from the strongly reactive strain $s e F_{5}$ are able to modify partially the level of reactivity of the females which have inherited them from their fathers.

The two experiments which have been reported bear out the same conclusion. The level of reactivity of any reactive female is controlled by several agents. By far, the most important ones are brought by the maternal gamete, but the chromosomes inherited through the paternal gamete are another certain, though minor, agent. When they originate from a weakly reactive strain, they tend to lower the level of reactivity and they act in the opposite way when they come from a strongly reactive strain. On the whole, the chromosomes behave as in a classical case of quantitative inheritance; they act even in the heterozygous state and their actions are additive. Nevertheless, the different chromosomes are not equivalent, some of them being undoubtedly particularly efficient in controlling reactivity.

\section{(iii) The effect on the level of reactivity of changing the genotype within the same maternal line}

Since, in the crosses between strong and weak reactive strains, the level of reactivity of a female depends to some extent on the chromosomes which she has inherited from her father, it was interesting to see what happens when, by successive backcrosses, a new strain is built which associates the homozygous genotype of a neutral strain with maternal derivation from a strong reactive one. The level of reactivity of such a composite strain will indicate how the chromosomes interact, in the long run, with the cytoplasm.

Females with the genotype $M-5-(r) /+-(n) ; C y-(r) / c n-(n) ; D c x F-(r) /+-(n)$ 
were obtained from the same initial crosses as in the experiment presented in the first part (fig. 1). These females were crossed with males of the neutral Paris strain and 53 daughters carrying no dominant mutation were collected (fig. 1b). Such females are derived in maternal line from the strongly reactive strain $s e F_{5}$ but have the genotype of the neutral Paris strain, except possibly for the chromosome 4 which was not checked. These females are referred to as Paris (cyt. seF $F_{5}$ ). They were crossed individually with two males of the inducer strain $L u$, and the fertility of a group of progeny from each of 53 crosses was measured. The 53 females were then crossed with their Paris (cyt. seF $F_{5}$ ) brothers. The latter cross permits conservation of Paris (cyt. $s e F_{5}$ ) families derived from a single female, the reactivity of which has been measured. The cinnabar mutation born by the Paris strain makes it possible to distinguish among the offspring of each female those which come from $L u$ males and those from Paris $\left(c y t . s e F_{5}\right)$ males.

Two other kinds of crosses were made as controls. Females of the $s e F_{5}$ and Paris strains were individually crossed with two $L u$ males and the fertility of progeny from each of the females was measured. The first cross enabled measurement of the level of reactivity of the $s e F_{5}$ strain and the second was a means of measuring the hatching percentage of eggs from normally fertile females, heterozygous for the chromosomes from Paris and $\mathrm{Lu}$ strains.

The results (fig. 7) show clearly that the progeny of crosses between Paris $\left(c y t . s e_{5}\right.$ ) females and $L u$ males are strongly sterile (fig. $7 \mathrm{a}$ ), while the control crosses between Paris females and $L u$ males give entirely fertile progeny (fig. 7c). This indicates that the Paris (cyt. seF $F_{5}$ ) females show a rather high reactivity which has been inherited from their maternal ancestors independently of the three major chromosomes of reactive origin. As in the experiments mentioned in section 2, it is not possible to assume that chromosome 4, which has not been controlled, is responsible for this hereditary transmission, since about only half of the Paris $\left(c y t . s e F_{5}\right)$ females are expected to have one of their chromosomes 4 of reactive origin.

Nevertheless, it should be noted that females originating from crosses between Paris (cyt. seF $F_{5}$ ) females and $L u$ males (fig. 7a) are, on average, less sterile than those from crosses between $s e F_{5}$ females and $L u$ males (fig. 7b). The reactivity of Paris ( $c y t . s e F_{5}$ ) females is therefore less strong than

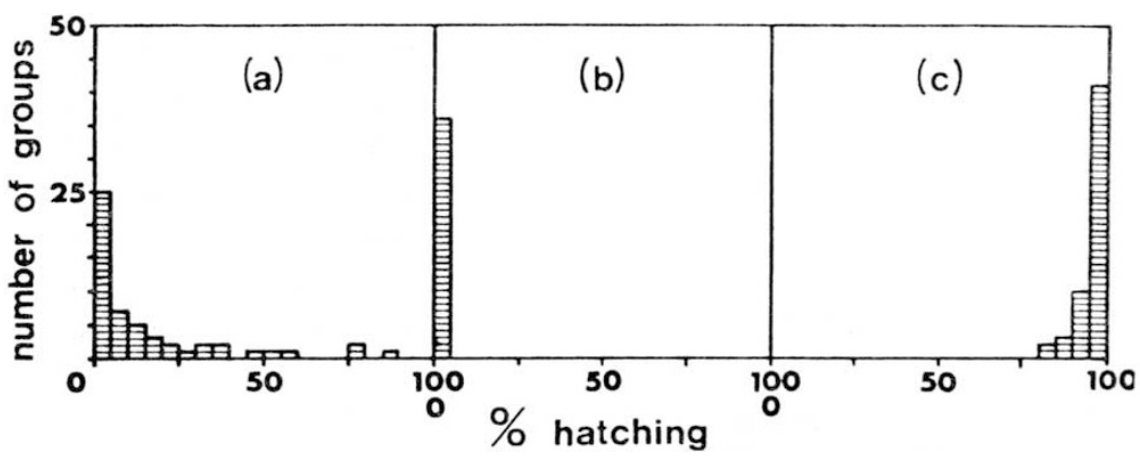

Frg. 7.-Effect on the level of reactivity of changing the genotype within the same maternal line. Each rectangle represents the fertility of a group of about 25 females. The three histograms represent the groups from crosses of two $L u$ males with single females of the strains Paris (cyt. seF $F_{5}$ ) (a), seF $F_{5}$ (b), Paris (c). 
that of $s e F_{5}$ females. As was already noted in the preceding experiments, the transmission of the level of reactivity is not entirely extrachromosomal. The chromosomes introduced by the two successive crosses to males of the neutral strain are effective in lowering this level. It was of course of interest to follow the evolution of the reactivity of Paris ( $\left.c y t . s e F_{5}\right)$ females through several generations.

Four Paris (cyt. se $F_{5}$ ) families, derived from four females of known reactivity, were maintained by successive endogamous crossing. Periodically, the level of reactivity of each family was measured in the following way: 10 females divided into two groups of five were crossed with males of the inducer strain $L u$, and the fertility of the descendants from each of these crosses was measured on a sample of eggs laid by about 20 females. As a control, for each of the four families, $10 \mathrm{Lu}$ females, divided into two groups of five, were crossed with Paris ( $c y t . s e F_{5}$ ) males and the fertility of progeny from each cross was measured.

\section{TABLE 3}

Evolution over several generations of the reactivity of $\mathrm{Paris}\left(\mathrm{cyt} . \mathrm{seF}_{5}\right.$ ) families. The table gives the hatching percentages of eggs laid by two groups of progeny from two crosses between five Paris (cyt. $\mathrm{seF}_{5}$ ) females belonging to the generation indicated and five $\mathrm{Lu}$ males. The generations are counted from when the Paris (cyt. $\mathrm{seF}_{5}$ ) files were obtained. Thus the females of the generation GO are the four females which gave rise to the four families, and the measure indicated is one of those represented in fig. 7a. The percentages are given with their standard error

\begin{tabular}{|c|c|c|c|c|}
\hline \multirow[b]{2}{*}{ Generations } & \multicolumn{4}{|c|}{ Families } \\
\hline & 1 & 2 & 3 & 4 \\
\hline 0 & $79 \cdot 5 \pm 3 \cdot 1$ & $54 \cdot 3 \pm 3 \cdot 3$ & $12 \cdot 1 \pm 1 \cdot 9$ & 0.0 \\
\hline 2 & $\begin{array}{l}56 \cdot 3 \pm 4 \cdot 4 \\
75 \cdot 6 \pm 3 \cdot 8\end{array}$ & $\begin{array}{l}77 \cdot 8 \pm 2 \cdot 5 \\
77 \cdot 1 \pm 3 \cdot 3\end{array}$ & $\begin{array}{l}17 \cdot 7 \pm 2 \cdot 2 \\
21 \cdot 5 \pm 2 \cdot 4\end{array}$ & $\begin{array}{l}16 \cdot 1 \pm 2.5 \\
11.9 \pm 1 \cdot 8\end{array}$ \\
\hline 4 & $\begin{array}{l}94 \cdot 9 \pm 1 \cdot 4 \\
72 \cdot 7 \pm 3 \cdot 1\end{array}$ & $\begin{array}{l}91 \cdot 3 \pm 1 \cdot 9 \\
77 \cdot 0 \pm 3 \cdot 8\end{array}$ & $\begin{array}{l}46 \cdot 3 \pm 3 \cdot 5 \\
51 \cdot 6 \pm 3 \cdot 4\end{array}$ & $\begin{array}{r}7 \cdot 6 \pm 1 \cdot 8 \\
17 \cdot 0 \pm 3 \cdot 8\end{array}$ \\
\hline 6 & $\begin{array}{l}88 \cdot 8 \pm 2 \cdot 2 \\
95 \cdot 9 \pm 2 \cdot 3\end{array}$ & $\begin{array}{l}88 \cdot 9 \pm 2 \cdot 0 \\
83 \cdot 6 \pm 2 \cdot 8\end{array}$ & $\begin{array}{l}91 \cdot 3 \pm 2 \cdot 3 \\
56 \cdot 0 \pm 4 \cdot 1\end{array}$ & $\begin{array}{l}82 \cdot 3 \pm 3 \cdot 0 \\
59 \cdot 0 \pm 4 \cdot 8\end{array}$ \\
\hline 8 & $\begin{array}{l}87 \cdot 5 \pm 2 \cdot 5 \\
79 \cdot 8 \pm 3 \cdot 1\end{array}$ & $\begin{array}{l}81 \cdot 4 \pm 3 \cdot 1 \\
87 \cdot 5 \pm 2 \cdot 4\end{array}$ & $\begin{array}{l}84 \cdot 5 \pm 2 \cdot 4 \\
80 \cdot 8 \pm 2 \cdot 6\end{array}$ & $\begin{array}{l}40 \cdot 1 \pm 3 \cdot 6 \\
46 \cdot 9 \pm 3 \cdot 5\end{array}$ \\
\hline 10 & $\begin{array}{l}89 \cdot 1 \pm 2 \cdot 5 \\
92 \cdot 1 \pm 1 \cdot 9\end{array}$ & $\begin{array}{l}96 \cdot 8 \pm 1 \cdot 3 \\
91 \cdot 7 \pm 2 \cdot 0\end{array}$ & $\begin{array}{l}75 \cdot 0 \pm 3 \cdot 6 \\
76 \cdot 1 \pm 3 \cdot 5\end{array}$ & $\begin{array}{l}75 \cdot 3 \pm 3 \cdot 6 \\
64 \cdot 4 \pm 3 \cdot 6\end{array}$ \\
\hline 35 & $\begin{array}{l}89 \cdot 6 \pm 2 \cdot 4 \\
94 \cdot 4 \pm 1 \cdot 6\end{array}$ & $\begin{array}{l}91 \cdot 8 \pm 1 \cdot 8 \\
91 \cdot 6 \pm 1 \cdot 8\end{array}$ & $\begin{array}{l}91 \cdot 7 \pm 1 \cdot 8 \\
87 \cdot 7 \pm 2 \cdot 2\end{array}$ & $\begin{array}{l}88 \cdot 3 \pm 2 \cdot 4 \\
89 \cdot 8 \pm 2 \cdot 3\end{array}$ \\
\hline Controls & $\begin{array}{l}95 \cdot 3 \pm 1 \cdot 5 \\
94 \cdot 2 \pm 1 \cdot 6\end{array}$ & $\begin{array}{l}94 \cdot 2 \pm 1 \cdot 6 \\
91 \cdot 6 \pm 2 \cdot 1\end{array}$ & $\begin{array}{r}93 \cdot 3 \pm 1 \cdot 8 \\
87 \cdot 7 \pm 2 \cdot 2\end{array}$ & $\begin{array}{l}88 \cdot 0 \pm 2 \cdot 4 \\
90 \cdot 8 \pm 1 \cdot 9\end{array}$ \\
\hline
\end{tabular}

The results are given in table 3 . In spite of the large variability which is observed within the families, it is clear that they all undergo an evolution in the same direction. With the families 1 and 2, which originated from relatively weakly reactive females, the hatching percentages become comparable with those of the controls after four to six generations. For the 
two other families, originating from more strongly reactive females, only the measurement of the 35th generation was similar to that of controls. Whatever the level of reactivity of the original female, after a variable length

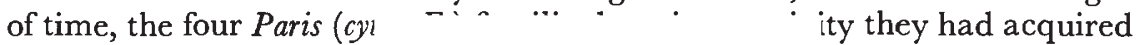
with the cytoplasm of the ser 5 snialerilal ancesturs anu become identical to the Paris strain whose genotype they contain. Therefore, it is clear that the action of the $-(n)$ chromosomes can accumulate through the generations and that, in the long run, it will eradicate the original maternal influence. A notable point is that this transformation requires more than 10 generations.

\section{Discussion}

Several conclusions can be drawn from the experimental results which have been presented:

(i) The amount of reduction of fertility of $S F$ females does not depend on the reactive or neutral origin of the chromosomes they received from their reactive mothers, but appears to be determined by a state which is established in the oocytes of these mothers.

(ii) Among the various reactive lines, this cytoplasmic state of the oocytes displays a large quantitative variation, which allows us to define what has been called the level of reactivity. The level of reactivity of any female can be measured by the amount of reduction of fertility observed in its daughters from a cross with standard inducer males.

The mode of inheritance of the level of reactivity was first investigated in studying females from crosses between strong and weak reactive strains. Among the females from any such cross, a rather large variation of levels of reactivity is observed, but most of them are quite similar to their mothers, and there is always a very important difference between the results of reciprocal crosses. The level of reactivity of a female is therefore chiefly determined by maternal inheritance, but depends to some extent on the father. It has been demonstrated that this paternal influence is linked to the three major chromosomes. The actions of the chromosomes are additive as in a classical case of quantitative inheritance.

It was further demonstrated that this action of the chromosomes can accumulate through the generations and finally eradicate any initial nonchromosomal maternal inheritance. Indeed, Paris ( $c y t . s e F_{5}$ ) families were built which descend in maternal line from the strongly reactive strain $s e F_{5}$, but have acquired by backcrosses the genotype of the neutral strain Paris. Initially strongly reactive, these families underwent a progressive evolution and, at the end, could not be distinguished from the neutral strain. This transformation was slow and required at least 10 generations for completion.

It should be noted that in an other experiment, the opposite evolution was observed (Pelisson, personal communication): a strain, derived in maternal line from a weak reactive strain, but having acquired the chromosomes of a strong reactive one, evolved slowly towards a high level of reactivity.

From this experimental evidence, it may be concluded that reactivity is the expression of a cytoplasmic state, displaying a large quantitative range of variation, which tends to be transmitted unchanged from mother to daughter. Nevertheless, it is influenced by a polygenic system borne by the chromosomes. In the long run, it is the chromosomal genotype which 
determines the level of reactivity, so there is no true permanent cytoplasmic inheritance of this character. A very important feature of the situation is the extremely slow rate with which the cytoplasmic state reacts to the influence of chromosomes. This might be explained by at least two different hypothesis:

1. The direct genetic determinants of reactivity may be extrachromosomal elements. They would form an intracellular population, which would tend to be regularly transmitted through mitosis, but would be liable to undergo quantitative or qualitative variations through the action of nuclear genes. In this hypothesis, the delayed character of the effect of a genotypic substitution is not very surprising.

2. The genetic determinants of reactivity could be strictly chromosomal. In order to explain the delayed effects observed, it might be assumed that the products of these genes exert a rather complex retroactivity on the expression of the genes themselves. As an alternative, the delayed effect might be due to the fact that the products of the genes involved in the control of levels of reactivity are incorporated in cellular membranes with modalities which are, to a large extent, fixed by the pre-existing structures. If this is the case, the situation would be comparable with the phenomena of structural inheritance (Whittle and Chen-Shan, 1972; Beisson et al., 1976).

The hereditary transmission of levels of reactivity shows some analogies with other well investigated genetic phenomena. Examples are the nuclear mutation affecting mitochondrial multiplication in Paramecium (Beisson et al., 1974; Sainsard-Chanet, 1976), or the expression of one of the mutants resistant to basic fushin in Saccharomyces cerevisiae (Barrere and Mounolou, 1976), where delayed effects of the genotype have been demonstrated.

On the other hand, the inheritance of reactivity bears some similarity with the killer phenomenon in yeast. At least 10 nuclear genes are necessary for maintenance or replication of the killer particles in Saccharomyces cerevisiae (Wickner and Leibowitz, 1976). In the same organism, the presence of virus-like particles of V2 type, which are implicated in the killer phenomenon, is always associated with the presence of virus-like particles of the VI type and of a particular allele of a nuclear gene (Mitchell et al., 1976). This situation approximates to the first hypothesis suggested above to explain the transmission of levels of reactivity.

Experiments are in progress to determine which of the two hypotheses suggested is the more likely.

Acknowledgments.-The authors express special thanks to Prof. J. C. Bregliano for advice throughout this work and for many helpful comments on the manuscript. They also thank Prof. Ph. L'Héritier for his critical reading of the manuscript. This work was supported by grants from the Centre National de la Recherche Scientifique (ERA 692: Phénomènes d'hérédité non mendélienne chez la Drosophile) and from the University of ClermontFerrand II.

\section{REFERENCES}

BARRERE, G., AND MOUNOLOU, J. C. 1976. Yeast mutants resistant to basic fuchsin: a genetic approach to the integration of nuclear and mitochondrial information. Mutation Research, 35, 39-52.

BeIsSON, J., LEFORT-TRAN, M., POUPhile, M., ROSSIGNOL, M., AND SATIR, B. 1976. Genetic analysis of membrane differentiation in Paramecium. Freeze fracture study of tricocyst cycle in wild type and mutant strains. J. Cell Biology, 69, 126-143. 
beisson, J., SAinsard, A., AND adoutte, A., beale, G. H., KnOWles, J., AND tait, A., 1974. Genetic control of mitochondria in Paramecium. Genetics, 78, 403-413.

Bucheton, A. 1973. Contribution à l'étude de la stérilité femelle non mendélienne chez Drosophila melanogaster. Transmission héréditaire des degrés d'efficacité du facteur " réacteur". Comptes Rendus Ac. Sc. Paris, D, 276, 641-644.

bucheton, A., LAvige, J. M., PicArd, G., AND L'héritier, PH. 1976. Non-Mendelian female sterility in Drosophila melanogaster : quantitative variations in the efficiency of inducer and reactive strains. Heredity, $36,305-314$.

DAvid, J. 1959. Etude quantitative du développement de la Drosophile élevée en milieu axénique. Bul. Soc. Biol. France et Belgique, 93, 4, 472.

keArsey, M. J., Williams, W. R., Allen, P., AND COUlter, F. 1977. Polymorphism for chromosomes capable of inducing female sterility in Drosophila. Heredity, 38, 109-115.

KIDWELl, M. G., KIDWell, J. F., AND sveD, J. A. 1977. Hybrid dysgenesis in Drosophila melanogaster: a syndrome of aberrant traits including mutation, sterility and male recombination. Genetics, $86,813-833$.

LINDSLEY, D. L., AND GRELL, E. H. 1967. Genetic variations of Drosophila melanogaster. Carnegie Institution of Washington Publication, 627.

mitchell, D. J., Herring, A. J., AND BEVAN, E. A. 1976. The genetic control of DS-RNA virus-like particles associated with Saccharomyces cerevisiae killer yeast. Heredity, 37 , 129-134.

PICARD, G. 1976. Non-mendelian female sterility in Drosophila melanogaster: hereditary transmission of $I$ factor. Genetics, 83, 107-123.

PICARD, G. 1977. Le phénomene $S F$ chez Drosophila melanogaster: mise en évidence des facteurs $R$ et $I$ et des principales proprietés de $I$. Thèse de Doctorat d'Etat, Université de Clermont-Ferrand II.

picard, G., Bucheton, A., LAvige, J. M., AND fleUriet, A. 1972a. Contribution à l'étude d'un phénomène de stérilité à déterminisme non mendélien chez Drosophila melanogaster. Comptes Rendus Ac. Sc. Paris, D, 275, 933-936.

piCARD, G., BUCheton, A., LAVige, J. M., Fleuriet, A., BREgliano, J. C., AND L'héRitier, PH. 1972b. Further data on non-mendelian female sterility in $D$. melanogaster. Dros. Inf. Serv., 49, 45.

PiCARD, G., BUCheton, A., LAvige, J. M., AND PELISSON, A. 1976. Répartition géographique des trois types de souches impliquées dans un phénomène de stérilité à déterminisme non mendélien chez Drosophila melanogaster. Comptes Rendus Ac. Sc. Paris, D, 282, 1813-1816.

PiCARD, G., LAVIGE, J. M., BUCheton, A., AND BREgliano, J. c. 1977. Non mendelian female sterility in Drosophila melanogaster: physiological pattern of embryo lethality. Biologie cellulaire 29, 89-98.

ROSENFELD, A., CARPENTER, A., AND SANDLER, L. 1971. A nonchromosomal factor causing female sterility in D. melanogaster. Dros. Inf. Serv., 47, 85.

SAINSARD-CHANET, A. 1976. Gene controlled selection of mitochondria in Paramecium. Molecular and General Genetics, 145, 23-30.

sved, J. A. 1976. Hybrid dysgenesis in Drosophila melanogaster: a possible explanation in terms of spatial organization of chromosomes. Aust. F. Biol. Sci., 29, 375-388.

WhitTle, J. R. s., AND ChEN-Shan, L. 1972. Cortical morphogenesis in Paramecium aurelia: mutants affecting cell shape. Genet. Res., 19, $271-279$.

WICKNER, R. B., AND LEIBOWITZ, M. J. 1976. Two chromosomal genes required for killing expression in killer strains of Saccharomyces cerevisiae. Genetics, 82, 429-442. 